\title{
Study on Design, Manufacture and Cutting Performance of Circular-arc Milling Cutters for Machining Titanium Alloy
}

\section{Tao Chen ( $\nabla$ dotnetchen@163.com )}

Harbin University of Science and Technology

\section{Liu Gang}

Harbin University of Science and Technology

Li Rui

Harbin University of Science and Technology

\section{Lu Yujiang}

Harbin University of Science and Technology

\section{Wang Guangyue}

Harbin University of Science and Technology

\section{Research Article}

Keywords: Titanium alloy TC4, Circular-arc milling cutter, Design and manufacture of milling cutters, Wear morphology, Surface quality

Posted Date: June 18th, 2021

DOI: https://doi.org/10.21203/rs.3.rs-621154/v1

License: (c) (1) This work is licensed under a Creative Commons Attribution 4.0 International License.

Read Full License 


\title{
Study on Design, Manufacture and Cutting Performance of Circular-arc Milling Cutters for Machining Titanium Alloy
}

\author{
Chen Tao*, Liu Gang, Li Rui, Lu Yujiang, Wang Guangyue
}

School of Mechanical and Power Engineering, Harbin University of Science and Technology, Harbin 150080, China

(*Corresponding author: dotnetchen@163.com)

\begin{abstract}
Titanium alloy is widely used for manufacturing structural parts of high-end equipment due to its excellent mechanical properties, despite difficulty in being machined. Nowadays, titanium alloy parts are mostly machined by ball-end milling cutters (BEMC), but the cutting edge structure of the BEMC limits the improvement in machining efficiency and surface quality of the parts. In this paper, a circular-arc milling cutter (CAMC) with large-curvature cutting edge was proposed; the differential geometry method was used for establishing the geometric model for the contour surface of the CAMC and the mathematical model for the spiral cutting edge line; the conversion matrix between grinding wheel and workpiece coordinates was introduced to derive the equation of grinding wheel trajectory when the rake face of the CAMC was ground; the self-designed CAMC was ground and tested in accuracy. The comparative research was conducted experimentally on the side milling of titanium alloy TC4 with the CAMC and BEMC, and consequently the variation laws of milling forces, wear morphology and machined surface quality were obtained about the two types of milling cutters. The results indicated that the CAMC can effectively reduce the main milling force and keep the milling process stable. Moreover, the CAMC was worn slower and produced better surface quality than the BEMC.
\end{abstract}

Keywords: Titanium alloy TC4, Circular-arc milling cutter, Design and manufacture of milling cutters, Wear morphology, Surface quality

\section{Introduction}

Titanium alloy has become preferable material for key parts in the aerospace industry due to its merits such as high strength, strong corrosion resistance and low-temperature performance $[1,2]$. Cutting tools meet new challenges as the manufacture of high-end equipments makes an increasing demand on the machining quality and efficiency of titanium alloy components. Nowadays, the frequently used ball-end milling cutters (BEMC) have large-curvature cutting edge, and thus tend to shrink milling step to ensure good machined surface quality, but this decreases the efficiency of machining titanium alloy and meanwhile increases the manufacturing cost more or less[3,4]. Therefore, it is essential and urgent to improve the efficiency of milling titanium alloy for its better application and promotion.

In research on the cutting performance and grinding of BEMC, Chen et al. [5] presented a new mathematical model for S-shaped edge curve. The model overcomes the complex computation and poor adaptability of the traditional modeling method, and enhances the cutting efficiency and stability of BEMC. Masahiko et al. [6] developed successfully two types of BEMC with a unique cutting edge and verified experimentally that a high finishing accuracy and minor cutting forces were realized by the above-mentioned cutting tools. Engin et al. [7] established a generalized mathematical model of most helical end milling cutters, and verified correctness of the model by the experiment on milling titanium alloy. Ren et al. [8] proposed a novel method of five-axis flute grinding based on the theories of analytic geometry and envelope, and verified the validity of the method by 3D simulations and machining experiments. The results show that this grinding method can effectively reduce the errors in 
grinding helical flutes. Pham et al. [9] proposed a manufacturing model of flat-end milling cutters using a five-axis computer numerical control grinding machine, based on the research into a given wheel profile and the relative movements between workpiece and grinding wheel. The model was practical and efficient for the manufacture of an end milling cutter. Wang et al. [10] generated a model of grinding wheel trajectory for machining helical grooves in terms of the grinding wheel's position and orientation, and provided a new approach for flute grinding of end milling cutters. Chen et al. [11] proposed new mathematical models and grinding methods of BEMC based on the orthogonal spiral cutting edge curve. It was found through the grinding experiments that both rake face with equal rake angle and flank face with equal clearance angle improved effectively chip removal conditions and machining performance of BEMC. Li et al. [12] reported a graphical analysis method to obtain the structural parameters and geometric shapes of helical grooves. Based on this, the influence of the geometry and grinding parameters of grinding wheels was discussed on radial rake angles, groove width and core radius. Tang et al. [13] proposed three practical and reliable grinding methods based on the research into mathematical models of spiral grooves, conical flank and cutter clearance, and verified experimentally reliability of the proposed grinding methods. Chen et al. [14] presented a novel method to grind the rake face of a taper BEMC using a CBN spherical grinding wheel. This method used the self-adaptation characteristics of a sphere to decrease the number of simultaneous cooperative axes of the $\mathrm{CNC}$ tool grinder from 5 to 4 . This boosted the grinding accuracy of rake faces. Sartori et al. [15] investigated the tool wear mechanism under different cooling conditions during machining titanium alloy, and found that cryogenic cooling conditions can effectively reduce adhesion and coating peeling. Krishnaraj et al. [16] researched surface roughness, cutting forces, and cutting temperature at different cutting parameters through the orthogonal experiment on machining titanium alloy, and revealed the relationship of cutting parameters with cutting temperature and surface quality. Shi et al. [17] carried out the experiments on milling titanium alloy TC21. Based on the experimental results, the tool materials and milling parameters were optimized and thus the tool life in milling titanium alloy TC21 was prolonged. Amin et al. [18] conducted a comparative experimental study on machining titanium alloy Ti-6Al-4V with polycrystalline diamond and uncoated tungsten carbide-cobalt inserts. The results indicated that the surface roughness machined by uncoated carbide tools increased with cutting speed. Yang et al. [19] carried out a study on the surface roughness in milling titanium alloy TC17 using carbide ball end mills, and found the surface roughness was optimal when the rotational angle ranged from $0^{\circ}$ to $90^{\circ}$ with a constant inclination angle. Su et al. [20] investigated the tool performance in high-speed milling of TA15 alloy using the new material tools, PCD and PCBN tools, and concluded that attrition, adhesion and diffusion were main wear mechanisms of PCD and PCBN tools in high-speed milling of TA15. Wu et al. [21] presented a systematic method for design and manufacture of circular-arc ball-end rotating cutters, and deduced the section profile and relative feeding speed of grinding wheels in machining processes. Moreover, the influence of grinding wheel position on manufacture of tools was analyzed according to the computer simulation result of groove surface enveloped by grinding wheel.

To sum up, the overall research into the milling cutters for machining titanium alloy mostly focuses on the optimization of tool geometry characteristics and the tool path planning for multi-axis machining center [22-24]. Nowadays some companies such as Walter and Fraisa have proposed a few novel cutting tools for machining titanium alloy. The novel cutters partly increase the curvature of side cutting edge through reducing the edge radius of ball end, and thus enhance side milling efficiency. Nonetheless, smaller radius of bottom edge increases grinding difficulty and decreases overall tool 
rigidity. Consequently, combined with the design idea for integration of tool design and manufacture [25], this paper employed the differential geometry method for designing a circular-arc milling cutter (CAMC) with large curvature, resolving the contradiction of the above novel cutters between edge radius of ball end and overall tool rigidity. This provides a new idea and method for the design and manufacture of milling cutters for machining titanium alloy.

\section{Design of CAMC}

\subsection{Presentation of tool structure and establishment of geometric model}

The CAMC designed in this paper is a rotary tool. The tool contour can be obtained by rotating the generatrix around the rotation axis. Figure 1 shows the generatrix of the CAMC in the $X_{0} O Z_{0}$ coordinates. The generatrix consists of end cutting edge $\mathrm{AB}$, transitional arc $\mathrm{BC}$ and circumferential cutting edge $C D$. The two parts of cutting edge are connected by the transitional arc. $R_{1}$ stands for the arc radius of generatrix $A B$ at the end cutting edge, and its circle center is expressed as $\mathrm{O}_{1}$. $\mathrm{R}_{2}$ stands for the arc radius of the generatrix at the circumferential cutting edge, and its circle center is expressed as $\mathrm{O}_{2}$. The radius of transitional cutting edge $\mathrm{BC}$ is expressed as $\mathrm{r}$, and its circle center $\mathrm{O}_{3}$. In order to ensure each section of the arc are tangent and the arc radius of the transitional cutting edge is reduced, the arc radius of circumferential cutting edge CD can be 4-12 times as long as that of the tool shank.
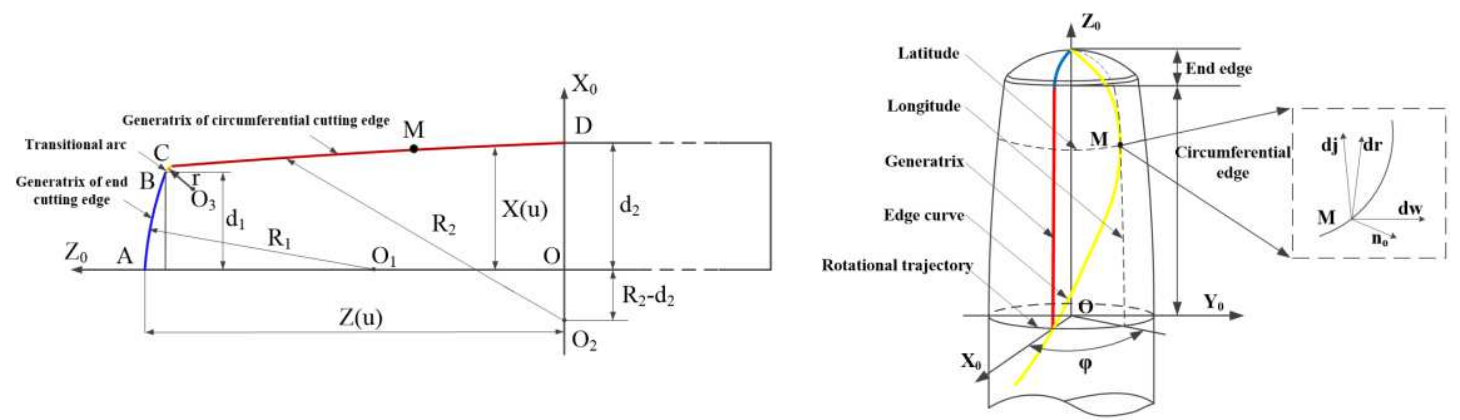

Figure 1. Generatrix of the CAMC.

Figure 2. Rotational contour of the CAMC.

Here the equation for the generatrix of end cutting edge $A B$ is expressed as follows.

$$
\left\{\begin{array}{l}
x(u)=R_{1} \sin \left(u / R_{1}\right) \\
z(u)=R_{2}-R_{1} \cos \left(u / R_{1}\right)
\end{array} \quad 0 \leq u \leq k_{1}\right.
$$

In the above equation, $\mathrm{u}$ stands for the arc length from starting point $\mathrm{A}$ to any point on the generatrix $\mathrm{AD}$ and has a value range of $0 \leq u \leq k_{1}$.

Here is the equation for the generatrix of circumferential cutting edge CD.

$$
\left\{\begin{aligned}
x(u)= & R_{2} \sin \left(s-k_{1}+k_{2} / R_{2}\right)-\left(R_{2}-d_{2}\right) \\
z(u)= & R_{2}-R_{1} \cos \left(\arcsin \left(d_{1} / R_{1}\right)\right) \quad k_{1} \leq u \leq k_{1}-k_{2}+0.25 \pi R_{2} \\
& \quad-R_{2} \cos \left(u-k_{1}+k_{2} / R_{2}\right)
\end{aligned}\right.
$$

$k_{1}$ and $k_{2}$ are respectively expressed as the following.

$$
\begin{aligned}
& k_{1}=R_{1} \arcsin \left(d_{1} / R_{1}\right) \\
& k_{2}=R_{2} \arcsin \left(\left(R_{2}+d 1-d 2\right) / R_{2}\right)
\end{aligned}
$$

Figure 2 displays the rotational contour of the CAMC in the coordinate system $O\left(X_{o} Y_{o} Z_{o}\right) . Z_{0}$ stands for the rotation axis. The equation for the rotational contour surface of the CAMC is expressed 
as follows.

$$
s_{m}=\left[\begin{array}{c}
x(u) \cos (\varphi) \\
x(u) \sin (\varphi) \\
z(u) \\
1
\end{array}\right]
$$

In the above equation, $\varphi$ refers to the angle rotating around $Z_{o}$ axis. The mathematical model for the structural contour surface of the CAMC was obtained by putting Eqs. (1) and (2) into Eq. (4). Here the mathematical model for the generatrix of end cutting edge $\mathrm{AC}$ goes as follows.

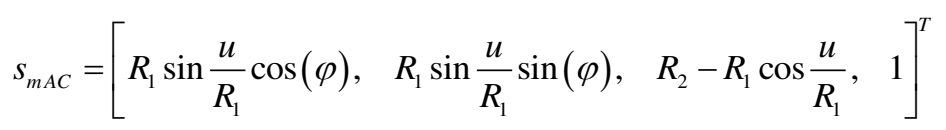

The mathematical model for the structural contour surface of circumferential cutting edge generatrix $\mathrm{CD}$ is expressed in the following.

$$
S_{m C D}=\left[\begin{array}{c}
{\left[R_{2} \sin \frac{u-k_{1}+k_{2}}{R_{2}}-\left(R_{2}-d_{2}\right)\right] \cos (\varphi)} \\
{\left[R_{2} \sin \frac{u-k_{1}+k_{2}}{R_{2}}-\left(R_{2}-d_{2}\right)\right] \sin (\varphi)} \\
R_{2}-R_{1} \cos \left(\arcsin d_{1} / R_{1}\right)-R_{2} \cos \frac{u-k_{1}+k_{2}}{R_{2}} \\
1
\end{array}\right]
$$

The lead of the equal-lead edge curve of the CAMC is expressed in $p$, and the relationship between lead $p$ and rotational angle $\varphi$ is shown in the following equation.

$$
p d \varphi=\frac{\partial r}{\partial u} d u
$$

The expression of the equal-lead edge curve of the CAMC was obtained by integrating Eq. (7), as shown below.

$$
\varphi(u)=\int \frac{1}{p} \sqrt{\left(x^{\prime}\right)^{2}+\left(z^{\prime}\right)^{2}} d u+\varphi c
$$

The equation for the edge curve of the CAMC was derived from simultaneous Eqs. (1), (2), (4), (5) and (8).

The equation for the end edge curve of the CAMC is expressed as follows.

$$
r_{A C}=\left[\begin{array}{c}
R_{1} \sin \left(u / R_{1}\right) \cos (u / p) \\
R_{1} \sin \left(u / R_{1}\right) \sin (u / p) \\
R_{2}-R_{1} \cos (u / p) \\
1
\end{array}\right]
$$

The following is the equation for the circumferential edge curve of the CAMC.

$$
r_{C D}=\left[\begin{array}{c}
D\left(1.4-C\left[\left(\lambda_{1}-s+0.1 L\right) / D\right]\right) C(s / p) \\
D\left(1.4-C\left[\left(\lambda_{1}-s+0.1 L\right) / D\right]\right) S(s / p) \\
0.6 L+D S\left[\left(\lambda_{1}-s+0.1 L\right) / D\right] \\
1
\end{array}\right]
$$

The arc radius $R_{l}$ of the generatrix of end cutting edge AC was set as $6 \mathrm{~mm}$ and $d_{1} 4 \mathrm{~mm}$; the arc radius $R_{2}$ of the generatrix of circumferential cutting edge CD was set as $118 \mathrm{~mm}$ and $d_{2} 5 \mathrm{~mm}$; lead $p$ 
was respectively set as $8 \mathrm{~mm}, 12 \mathrm{~mm}$, and $16 \mathrm{~mm}$. Then the numerical simulation was conducted based on the above value setting. The simulation results are shown in Figure 3.

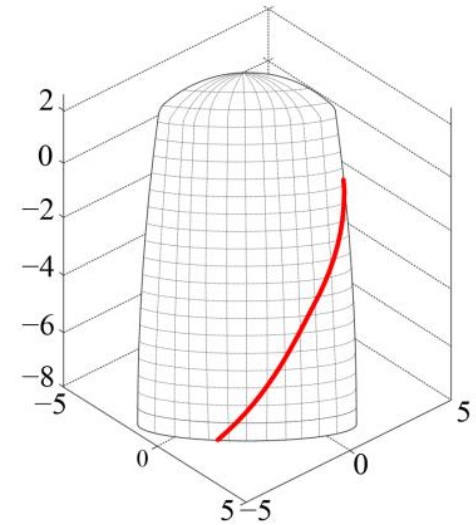

(a) $p=8 \mathrm{~mm}$

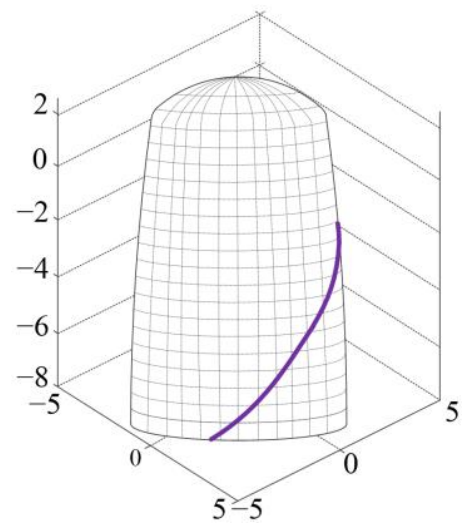

(b) $p=12 \mathrm{~mm}$

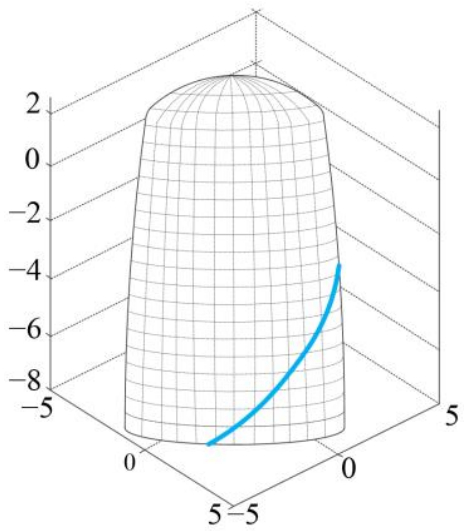

(c) $p=16 \mathrm{~mm}$

Figure 3. Simulation of the equal-lead edge curve of the CAMC.

\subsection{Model for rake faces of the CAMC}

Figure 4 shows rake and flank faces of the CAMC. In the figure, the base plane is set as $P_{r}$, the normal plane $P_{n}$, and the cutting plane $P_{s}$. The normal rake angle $\gamma_{n}$ is defined as the angle between rake face $P_{f}$ and base plane $P_{r}$, measured on the normal plane $P_{n}$. $N_{f}\left(n_{1}, n_{2}, n_{3}\right)$ stands for the unit normal vector of the rake face, and $N_{b}\left(n_{4}, n_{5}, n_{6}\right)$ the one of the flank face.

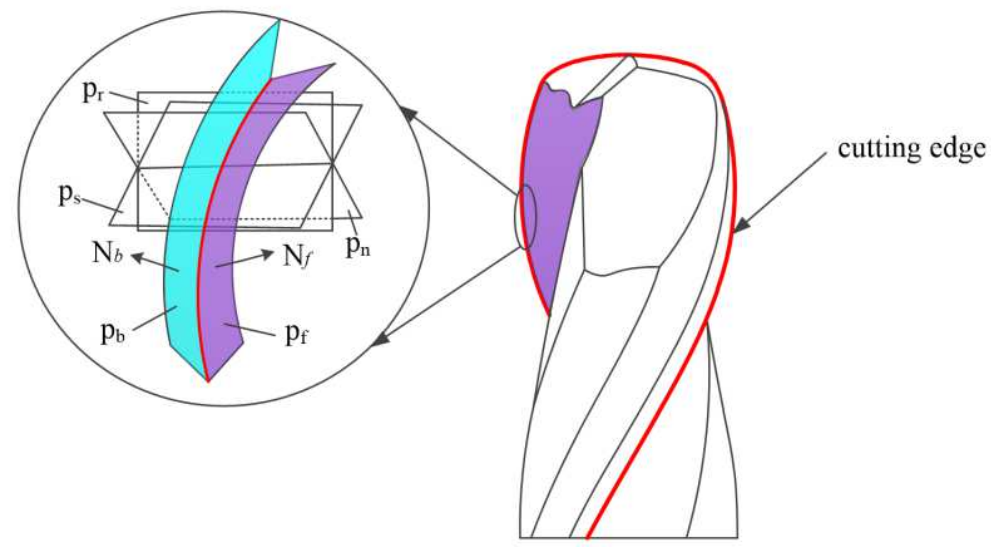

Figure 4. Rake and flank faces of the CAMC.

The following equation was derived from the geometric angle relationship between the rake face and the base plane of the CAMC.

$$
\begin{aligned}
& \frac{1}{\sqrt{1+\left(\frac{R_{1}}{p}\right)^{2} \sin \left(\frac{u}{R_{1}}\right)^{2}}}\left\{n_{1}\left[\cos \left(\frac{u}{R_{1}}\right) \cos \left(\frac{u}{p}\right)-\frac{R_{1}}{p} \sin \left(\frac{u}{R_{1}}\right) \sin \left(\frac{u}{p}\right)\right]\right. \\
& \left.+n_{2}\left[\cos \left(\frac{u}{R_{1}}\right) \sin \left(\frac{u}{p}\right)+\frac{R_{1}}{p} \sin \left(\frac{u}{R_{1}}\right) \cos \left(\frac{u}{p}\right)\right]+n_{3} \sin \left(\frac{u}{R_{1}}\right)\right\}=0
\end{aligned}
$$




$$
\begin{gathered}
\cos \left(\gamma_{n}\right)=-N_{f} \mathrm{~g}_{r} \\
=\frac{1}{\sqrt{1+\left[\frac{R_{1}}{p} \sin \left(\frac{u}{R_{1}}\right)\right]^{2}}}\left\{-n_{1}\left[\sin \left(\frac{u}{p}\right)-\frac{R_{1}}{p} \sin \left(\frac{u}{R_{1}}\right) \cos \left(\frac{u}{R_{1}}\right) \cos \left(\frac{u}{p}\right)\right]\right. \\
\left.+n_{2}\left[\cos \left(\frac{u}{p}\right)-\left(\frac{R_{1}}{p}\right) \sin \left(\frac{u}{R_{1}}\right) \cos \left(\frac{u}{R_{1}}\right) \sin \left(\frac{u}{p}\right)\right]-n_{3} \frac{R_{1}}{p} \sin ^{2}\left(\frac{u}{R_{1}}\right)\right\} \\
n_{1}{ }^{2}+n_{2}{ }^{2}+n_{3}{ }^{2}=1
\end{gathered}
$$

The normal vector of the rake face $N_{f}\left(n_{1}, n_{2}, n_{3}\right)$ was obtained by solving simultaneous equations (10), (11) and (12).

\section{Manufacture and detection of the CAMC}

\subsection{Establishment of grinding model for the CAMC}

For establishment of the mathematical model for the grinding trajectory of the CAMC, the coordinates of any point in the grinding wheel coordinate system needed to be converted to the workpiece coordinate system. Then the normal vector of the central point in grinding wheel was derived, based on the equations for edge curve as well as the relative position relationship between grinding wheel and workpiece. Furthermore, the mathematical model for grinding wheel position was deduced in milling. As is shown in Figure 5, in any position, the grinding wheel coordinate system $O_{g}-X_{g} Y_{g} Z_{g}$ was first moved parallel along the $X_{g}, Y_{g}, Z_{g}$ axes, and then rotated respectively around the $X_{g}, Y_{g}, Z_{g}$ axes. Here the translational distance is respectively expressed as $a_{x}, a_{y}, a_{z}$ and the rotational angles $\varphi_{x}, \varphi_{y}, \varphi_{z}$.

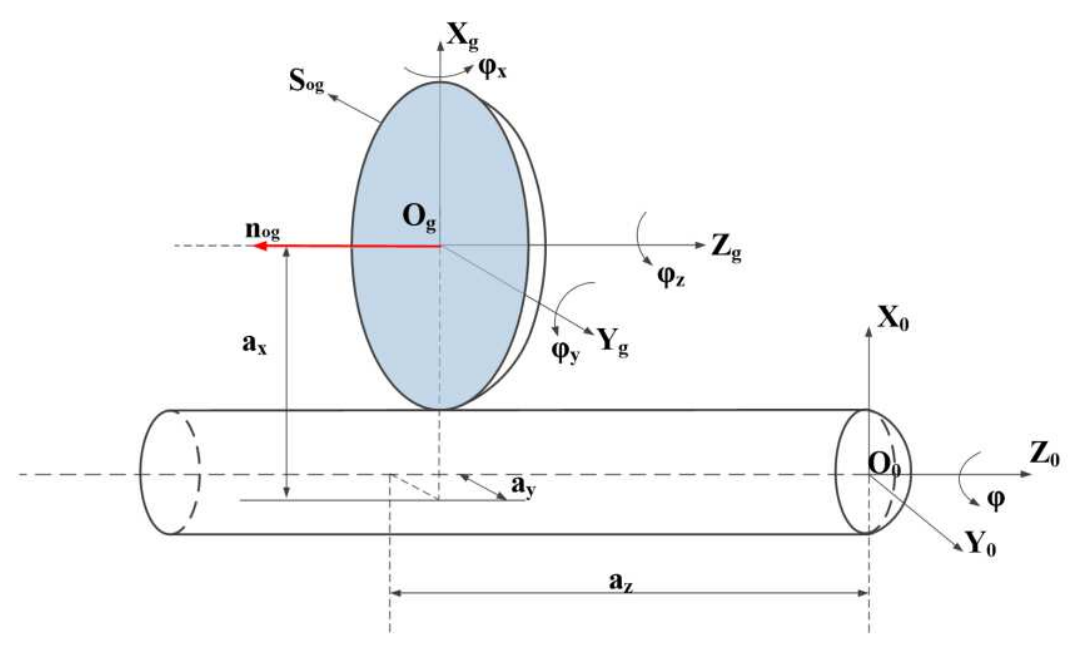

Figure 5. Conversion from grinding wheel to workpiece coordinate systems.

The conversion matrix from grinding wheel to workpiece coordinates is expressed as follows.

$$
\begin{aligned}
{ }^{o} A_{g} & =\operatorname{Rot}\left(Z_{g}, \varphi\right) \operatorname{Trans}\left(a_{x}, a_{y}, a_{z}\right) \operatorname{RPY}\left(\varphi_{x}, \varphi_{y}, \varphi_{z}\right) \\
& =\left[\begin{array}{cccc}
\Delta_{1} C \varphi_{y} & \Delta_{1} S \varphi_{y} S \varphi_{x}-\Delta_{2} C \varphi_{x} & \Delta_{1} S \varphi_{y} C \varphi_{x}+\Delta_{2} S \varphi_{x} & \operatorname{ax} C \varphi-a y S \varphi \\
\Delta_{2} C \varphi_{y} & \Delta_{1} S \varphi_{y} S \varphi_{x}+\Delta_{1} C \varphi_{x} & \Delta_{2} S \varphi_{y} C \varphi_{x}-\Delta_{1} S \varphi_{x} & \operatorname{ax} S \varphi+a y C \varphi \\
-S \varphi_{y} & C \varphi_{z} S \varphi_{x} & C \varphi_{z} C \varphi_{x} & a z \\
0 & 0 & 0 & 1
\end{array}\right]
\end{aligned}
$$

In the above equation, $S, C, \Delta_{1}$ and $\Delta_{2}$ are respectively abbreviations for $\sin (\varphi), \cos (\varphi), \cos \left(\varphi+\varphi_{z}\right)$, $\sin \left(\varphi+\varphi_{z}\right)$.

The coordinates of the rotational contour surface of grinding wheel were put into the conversion matrix for calculation. For convenience of calculation, the fourth line of the position matrix was set as 
1 , and then the position matrix of the rotational contour surface of grinding wheel was derived in the workpiece coordinates, as shown below.

$$
s_{o g}={ }^{o} A_{g} \cdot\left[\begin{array}{c}
x(h) \cos (v) \\
x(h) \sin (v) \\
z(h) \\
1
\end{array}\right]=\left[\begin{array}{l}
k_{3} C \varphi_{y} C \varphi+k_{4}\left[S \varphi_{y} S \varphi_{x} C \varphi-C \varphi_{x} S \varphi\right]+ \\
k_{5}\left[S \varphi_{y} C \varphi_{x} C \varphi+S \varphi_{x} S \varphi\right]+a_{x} C \varphi-a_{y} S \varphi, \\
k_{3} C \varphi_{y} S \varphi+k_{4}\left[S \varphi_{y} S \varphi_{x} S \varphi+C \varphi_{x} C \varphi\right]+ \\
k_{5}\left[S \varphi_{y} C \varphi_{x} S \varphi-S \varphi_{x} C \varphi\right]+a_{x} S \varphi+a_{y} C \varphi, \\
-k_{3} C \varphi_{y}+k_{4} C \varphi_{y} S \varphi_{x}+k_{5} C \varphi_{y} C \varphi_{x}+a_{z}, \\
1
\end{array}\right]
$$

In the equation, $h$ stands for the length of the grinding wheel generatrix and $v$ the angle of the grinding wheel generatrix rotating around the $Z_{g}$ axis. $k_{3}, k_{4}, k_{5}$ are all relevant parameters of the rotational surface of grinding wheel. Their expressions are specifically as follows.

$$
k_{3}=x(h) \cos (v) \quad k_{4}=x(h) \sin (v) \quad k_{5}=z(h)
$$

The unit normal vector $n_{o g}$ of the central point $O_{g}$ in grinding wheel is expressed in the workpiece coordinates as follows.

$$
\begin{aligned}
n_{o g}={ }^{o} A_{g} \cdot \frac{1}{\sqrt{x^{\prime}(h)^{2}+z^{\prime}(h)^{2}}}\left[\begin{array}{c}
z^{\prime}(h) \cos (v) \\
z^{\prime}(h) \sin (v) \\
-x^{\prime}(h) \\
0
\end{array}\right] \\
=\left[\begin{array}{l}
-z^{\prime}(h) C(v) C \varphi_{y} \Delta_{1}-z^{\prime}(h) S(v)\left[S \varphi_{y} S \varphi_{x} \Delta_{1}-C \varphi_{x} \Delta_{2}\right] \\
+x^{\prime}(h)\left[S \varphi_{y} S \varphi_{x} \Delta_{1}+S \varphi_{x} \Delta_{2}\right], \\
-z^{\prime}(h) C(v) C \varphi_{y} \Delta_{2}-z^{\prime}(h) S(v)\left[S \varphi_{y} S \varphi_{x} \Delta_{1}-C \varphi_{x} \Delta_{2}\right] \\
+x^{\prime}(h)\left[S \varphi_{y} C \varphi_{x} \Delta_{2}-S \varphi_{x} \Delta_{1}\right], \\
z^{\prime}(h) C(v) S \varphi_{y}-z^{\prime}(h) S(v) C \varphi_{y} S \varphi_{x}+x^{\prime}(h) C \varphi_{y} C \varphi_{x}, \\
0
\end{array}\right]
\end{aligned}
$$

Based on the simultaneous solution for the rotational surface matrix of grinding wheel (Eq. (15)) and the structural contour surface equations of tools (Eqs. (5), (6)) in the workpiece coordinates, the grinding wheel position equation was derived during grinding the rake face of the CAMC, as shown in the following.

$$
\begin{aligned}
R_{1} S\left(\frac{u}{R_{1}}\right) C\left(\frac{u}{R_{1}}\right) & =k_{3} C \varphi_{y} C \varphi+k_{4}\left[S \varphi_{y} S \varphi_{x} C \varphi-C \varphi_{x} S \varphi\right] \\
& +k_{5}\left[S \varphi_{y} C \varphi_{x} C \varphi+S \varphi_{x} S \varphi\right]+a_{x} C \varphi-a_{y} S \varphi \\
R_{1} S\left(\frac{u}{R_{1}}\right) S\left(\frac{u}{R_{1}}\right) & =k_{3} C \varphi_{y} S \varphi+k_{4}\left[S \varphi_{y} S \varphi_{x} S \varphi+C \varphi_{x} C \varphi\right] \\
& +k_{5}\left[S \varphi_{y} C \varphi_{x} S \varphi-S \varphi_{x} C \varphi\right]+a_{x} S \varphi+a_{y} C \varphi \\
R_{2}-R_{1} C\left(\frac{u}{R_{1}}\right) & =-k_{3} C \varphi_{y}+k_{4} C \varphi_{y} S \varphi_{x}+k_{5} C \varphi_{y} C \varphi_{x}+a_{z}
\end{aligned}
$$

The normal vector of the rotational surface of grinding wheel $n_{o g}$, the equation for the rotational contour surface of the tool generatrix, and the grinding wheel position equations (Eqs.17-19) were simultaneously solved, and thus the expressions for the normal vector of the rotational surface of grinding wheel were derived during grinding the rake face of the CAMC as follows. 


$$
\begin{gathered}
n_{1}(u)=\frac{-z^{\prime}(h) C(v) C \varphi_{y} C \varphi-z^{\prime}(h) S(v)\left[S \varphi_{y} S \varphi_{x} C \varphi-C \varphi_{x} S \varphi\right]+x^{\prime}(h)\left[S \varphi_{y} S \varphi_{x} C \varphi+S \varphi_{x} S \varphi\right]}{\sqrt{x^{\prime}(h)^{2}+z^{\prime}(h)^{2}}} \\
n_{2}(u)=\frac{-z^{\prime}(h) C(v) C \varphi_{y} S \varphi-z^{\prime}(h) S(v)\left[S \varphi_{y} S \varphi_{x} C \varphi-C \varphi_{x} S \varphi\right]+x^{\prime}(h)\left[S \varphi_{y} C \varphi_{x} S \varphi-S \varphi_{x} C \varphi\right]}{\sqrt{x^{\prime}(h)^{2}+z^{\prime}(h)^{2}}} \\
n_{3}(u)=\frac{z^{\prime}(h) C(v) S \varphi_{y}-z^{\prime}(h) S(v) C \varphi_{y} S \varphi_{x}+x^{\prime}(h) C \varphi_{y} C \varphi_{x}}{\sqrt{x^{\prime}(h)^{2}+z^{\prime}(h)^{2}}}
\end{gathered}
$$

In grinding the milling cutter, in order to ensure that the grinding wheel was in contact with the workpiece, the relative radial velocity at the contact point was kept 0 . Thus, the equation for the relative velocity at the contact point was obtained by differentiating the $t$ time on the position matrix of the rotational surface of grinding wheel (Eq.(15)), as is shown in the following.

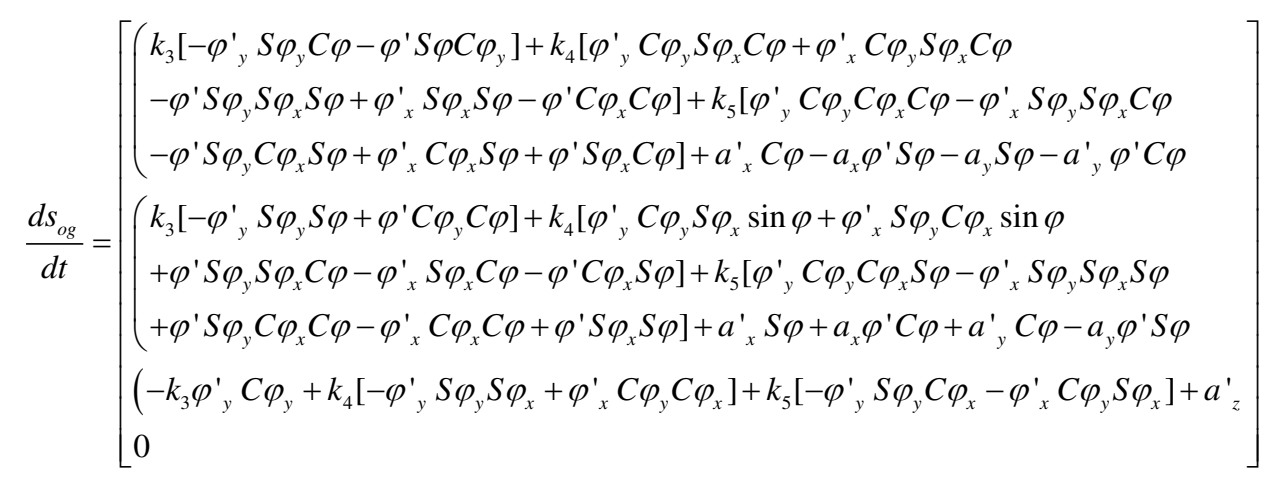

The equations for the circumferential and end edge curves of the CAMC were put into the above equation set and solved, and thus the mathematical models for the grinding wheel position $a_{x}, a_{y}, a_{z}, \varphi_{x}$, $\varphi_{y}$ and $\varphi$ were derived in grinding the rake face of the CAMC.

\subsection{Manufacture and detection of the CAMC}

The CAMC was ground on the SAACKE UWI five-axis grinding center, and the D64 flat grinding wheel and the 11V9 bowl-shape grinding wheel were used. The tool bar material is the K88UF carbide tungsten steel, its Rockwell hardness $91.7 \mathrm{HRA}$ and the particle size $0.8 \mu \mathrm{m}$. The grinding site of the CAMC is shown in Figure 6.

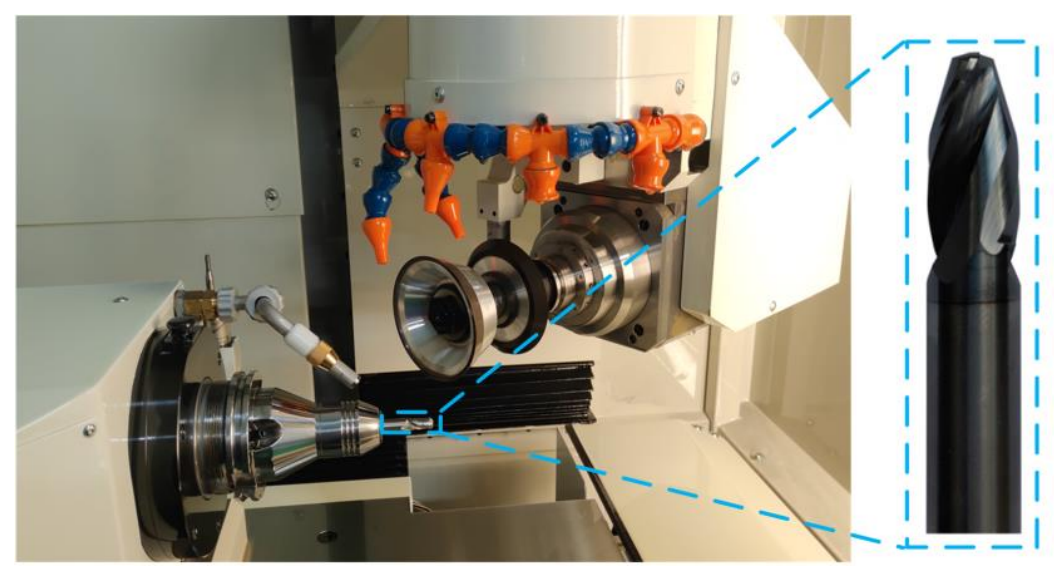

Figure 6. Grinding site of the CAMC.

In order to ensure the cutting performance for the use requirements, the manufactured CAMC 
must be detected in the dimensional and angular accuracy. The Genius 3 tool test instrument made in ZOLLER company was used for measuring the accuracy, meeting the demand for the dimensional tolerance less than $0.01 \mathrm{~mm}$ and the angular tolerance less than $0.5^{\circ}$.

Figure 7 shows the site and results of detecting the contour accuracy of the machined CAMC by the tool test instrument. The red lines represent the theoretical contour and the blue ones the grinding tolerance zone in the figure. The test results of grinding accuracy show that the grinding accuracy of the circumferential edge contour of the CAMC met the requirements, and there were minor errors in the upper end cutting edge and the transitional arc areas within the tolerance zone, so no impact was exerted on the normal use of the cutter. Furthermore the derived equation for grinding the CAMC in this paper can meet the requirement for the accuracy range in practical machining, so the equation was proved correct and can be used for manufacturing cutting tools.

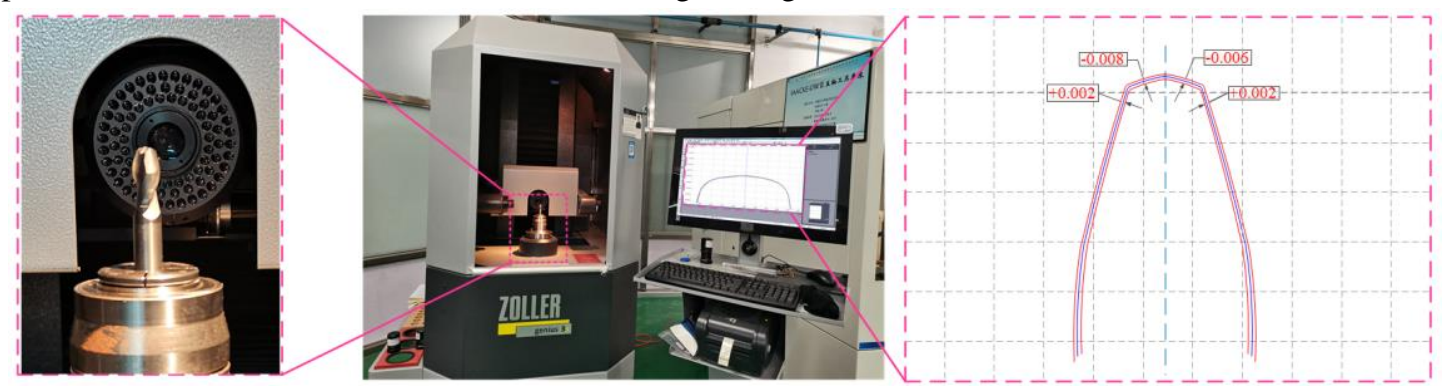

Figure 7. Site and results of detecting the contour accuracy of the machined CAMC.

\section{Comparative experiments on side milling of TC4}

\subsection{Experimental design}

Aiming to evaluate the cutting performance of the CAMC, this paper designed comparative experiments on side milling of TC4 titanium alloy using the BEMC and CAMC, and the experimental site is shown in Figure 8(a). The VDL-1000E three-axis milling center was selected as the machine tool. The two types of milling cutters for the experiments differed only in contour while they were the same in other geometric parameters. Namely, the diameters were both $10 \mathrm{~mm}$, the helical angles $30^{\circ}$, the rake angles $6^{\circ}$, the flank angles $12^{\circ}$, and the edge number 2 . The tool material was cemented carbide tungsten steel, the coating material $\mathrm{AlCrN}$ and the workpiece material TC4 titanium alloy with a dimension of $150 \mathrm{~mm} \times 100 \mathrm{~mm} \times 50 \mathrm{~mm}$. In the cutting parameters, the milling velocity was $70 \mathrm{~m} / \mathrm{min}$, the feed per tooth $0.06 \mathrm{~mm} / \mathrm{z}$, the milling depth $0.2 \mathrm{~mm}$, and the milling width $0.3 \mathrm{~mm}$.

The CAMC and BEMC are mostly used for machining inclined end faces of titanium alloy components, and thus the workpiece surface to be machined was first made into an inclined plane at an angle of $80^{\circ}$ to the horizontal plane before the experiment. Then the two types of milling cutters were used for side milling of the inclined plane. The specific milling method is shown in Figure 8(b).
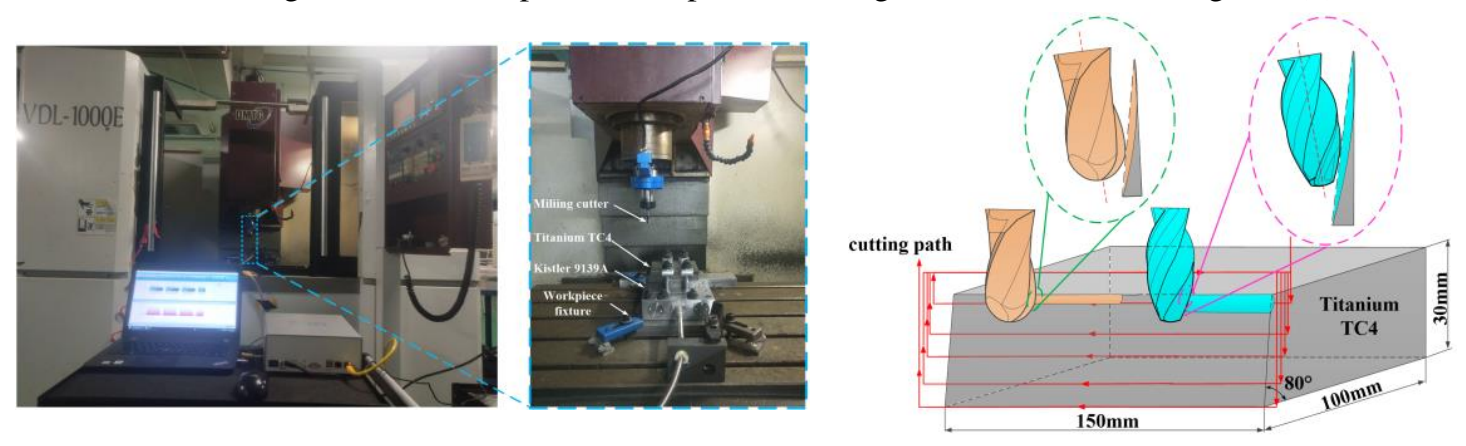
Figure 8. Experimental system and milling method.

The Kistler 9139A three-component piezoelectric dynamometer was used in the experiments to collect milling forces. The milling cutter was removed after milling every $3 \mathrm{~m}$, and then the VHX-1000 ultra-depth three-dimensional microscope and the SU3500 scanning electron microscope were used to observe and detect the tool wear morphology. Afterwards the workpiece was removed, and then the Taylor Surface CCI white light interferometer was used for observing the surface morphology of the machined workpiece.

\subsection{Analysis of cutting performance of the CAMC}

Figure 9 shows the comparison of the unworn CAMC and BEMC in milling forces and chip morphology. It is observed from the figure that the tangential force generated by the BEMC was obviously more than that by the CAMC, while the peak values of radial forces were close to each other. This is because the CAMC has a cutting edge with larger curvature and this decomposed effectively the milling forces in the tangential and axial directions, naturally reducing the radial force. Based on the overall change tendency for the chip morphology and milling forces, the tangential and radial forces generated by the CAMC were more stable than the ones by the BEMC, and the chips coming from the former were less serrated. The reason for the above is that larger curvature of the CAMC cutting edge causes longer cutting edge involved in cutting, and chip thinning makes heat dissipation easier so that chips are easier to be removed, and thus milling forces are more stable.

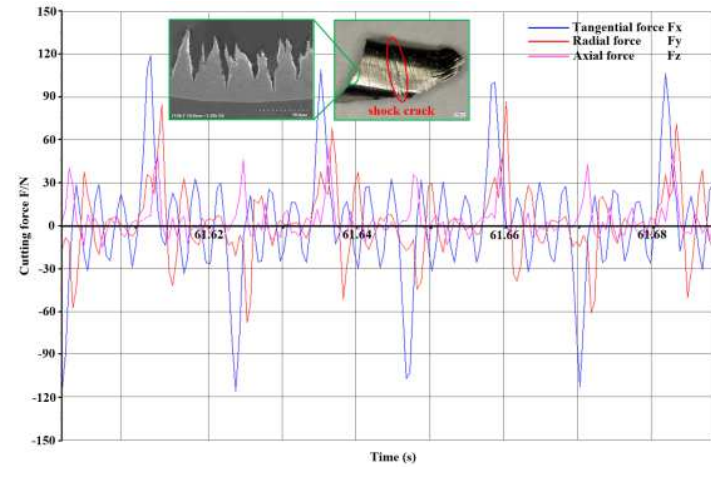

(a) BEMC

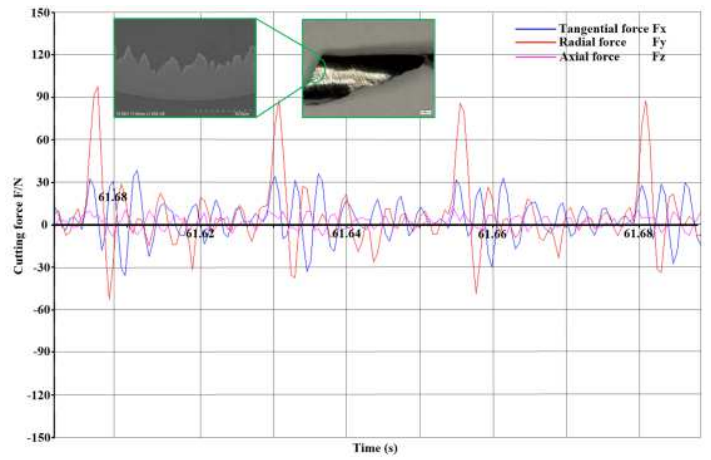

(b) CAMC

Figure 9. Milling forces and chip morphology generated by the BEMC and CAMC.

Figure 10 shows the comparison of the two milling cutters in flank wear micromorphology and machined surface quality. At the milling length of $60 \mathrm{~m}$, adhesion occurred on both flank faces, while a certain amount of coating peeling and tool tipping happened to the BEMC, causing poor surface quality of the machined workpiece. Compared with the BEMC, the CAMC showed no obvious tool tipping and realized relatively flat machined surface at the milling length of $120 \mathrm{~m}$. Furthermore, the energy spectrum analysis was conducted of both milling cutters. Some elements such as O, W, Co and Ti were found in Zone A of the BEMC, indicating that some diffusion wear occurred, while only the elements of $\mathrm{Ti}, \mathrm{Al}$, etc. from the workpiece were found on the flank face of the CAMC, showing that only coating peeling appeared. Here it was found that the workpiece surface machined by the BEMC was characterized by a large fluctuation in peak and trough, and the root mean square deviation of surface contour height arithmetic mean deviation of the surface contour height $S_{a}$ was larger than that by the CAMC. 


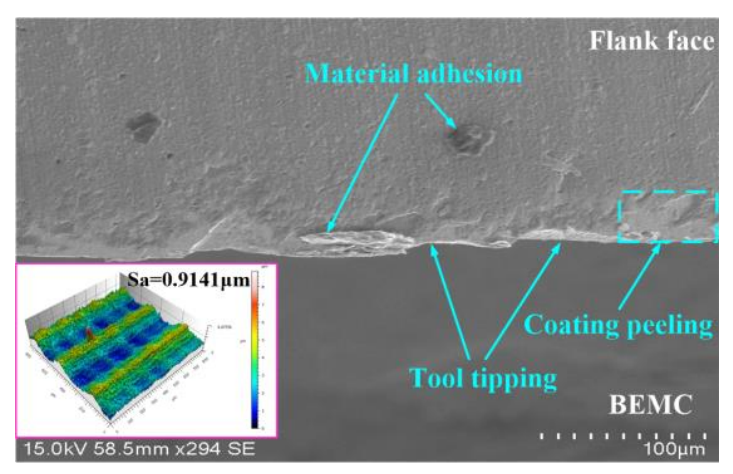

(a) At the milling length of $60 \mathrm{~m}$

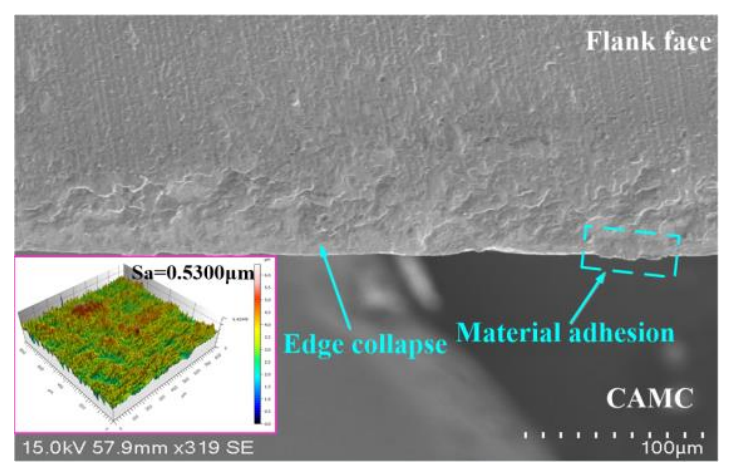

(c) At the milling length of $60 \mathrm{~m}$

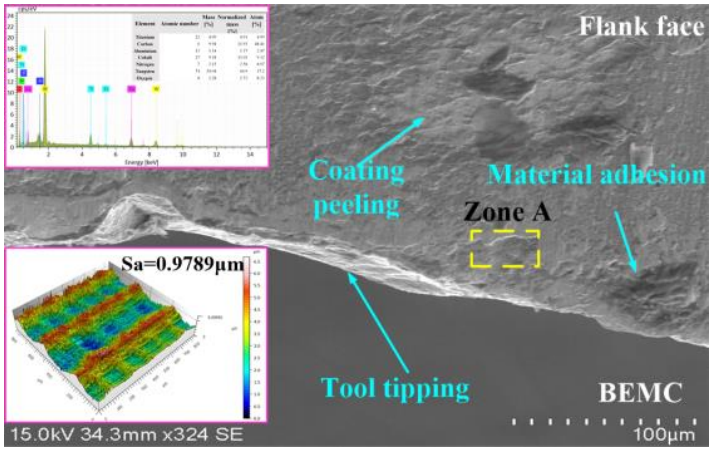

(b) At the milling length of $120 \mathrm{~m}$

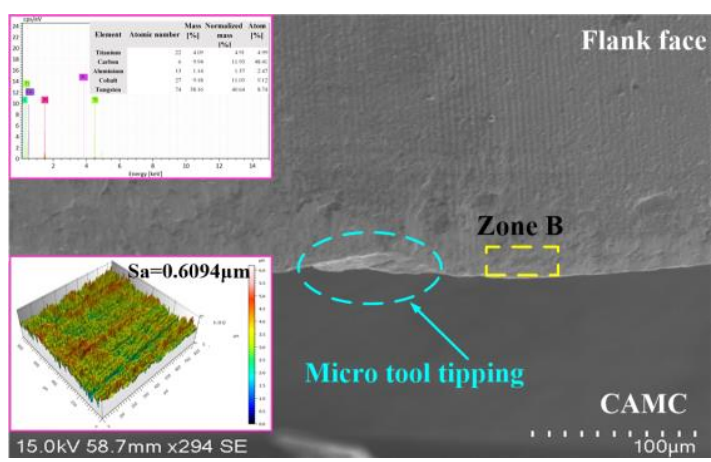

(d) At the milling length of $120 \mathrm{~m}$

Figure 10. Flank wear micromorphology and machined surface quality of BEMC and CAMC.

\section{Conclusion}

In this paper, the research was conducted on design, manufacture and cutting performance of circular-arc milling cutters (CAMC) for machining titanium alloy. Based on the cutting experiments, a comparative analysis was made of milling forces, chip morphology, tool wear and machined surface quality in milling titanium alloy with the CAMC and BEMC. The conclusions were drawn as follows.

(1) The research on design of the CAMC was carried out. The contour equations for the end and circumferential cutting edges of the CAMC were derived by the differential geometry method, and on this basis the mathematical model for the equal-lead helical edge line of the CAMC was established, and verified by MATLAB.

(2) The conversion matrix between grinding wheel and workpiece coordinates was derived, and the mathematical model for the rake face grinding trajectory of the CAMC was established. Grinding the CAMC and testing its geometric accuracy has realized evaluation for the grinding accuracy of developed cutting tools.

(3) Compared with the BEMC, the CAMC shows greater curvature of cutting edge, making the tangential and radial forces more stable in machining. According to the chip morphology, the chips generated by the CAMC are less serrated than the ones by the BEMC, and the unit chip shows no large deformation so that the chip removal is easier.

(4) By contrast with the BEMC, the CAMC is worn slower, shows no diffusion wear, and keeps better cutting performance. With increase of milling length, the residual height and roughness of the workpiece surface machined by the CAMC are still at a low level, and the surface contour changes slowly in shape and displays no obvious defects. 
Ethical Approval The research does not involve human participants or animals and the authors warrant that the paper fulfills the ethical standards of the journal.

Consent to Participate It is confirmed that all the authors are aware and satisfied of the authorship order and correspondence of the paper.

Consent to Publish All the authors are satisfied that the last revised version of the paper is published without any change.

Author contributions, Chen Tao, has organized the project, designed the cutting tools, and written the manuscript; Liu Gang, has designed the cutting tools, and written the manuscript; Li Rui, has conducted the experiments, and collected and analyzed data; Lu Yujiang, has manufactured and detected the cutting tools; Wang Guangyue, has reviewed the manuscript.

Funding This work was financially supported by the National Natural Science Foundation of China (Grant No. 51975168).

Competing Interests The authors declare that they have no competing interests.

Availability of data and materials The raw/processed data required to reproduce these findings cannot be shared for the time being. Data will be made available upon request.

\section{References}

[1] Pramanik A (2014) Problems and solutions in machining of titanium alloys. Int J Adv Manuf Tech, 70(5-8):919-928.

[2] Hou J, Zhou W, Duan H, Yang G, Xu H, Zhao N (2014) Influence of cutting speed on cutting force, flank temperature, and tool wear in end milling of Ti-6Al-4V alloy. Int J Adv Manuf Tech, 70(9-12):1835-1845.

[3] Wang ZG, Wong YS, Rahman M (2005) High-speed milling of titanium alloys using binderless CBN tools. Int J Mach Tool Manu, 45(1):105-114.

[4] Hsu CY, Huang CK, Wu CY (2007) Milling of MAR-M247 nickel-based super alloy with high temperature and ultrasonic aiding. Int J Adv Manuf Tech, 34:857-866.

[5] Cheng XF, Ding GF, Li R, Ma X, Qin S, Song X (2014) A new design and grinding algorithm for ball-end milling cutter with tooth offset center. P I Mech Eng B-J Eng, 228(7):687-697.

[6] Masahiko J, Isamu G, Takeshi W, Jun-ichi K, Masao M (2007) Development of CBN ball-nosed end mill with newly designed cutting edge. J Mater Process Tech, 192-193(1):48-54.

[7] Engin S, Altina Y (2009) Mechanics and dynamics of general milling cutters: part I: helical end mills. Int J Mach Tool Manu, (41):2195-2212.

[8] Ren L, Wang SL, Yi LL, Sun SL (2015) An accurate method for five-axis flute grinding in cylindrical end-mills using standard 1V1/1A1 grinding wheels. Precis Eng, 43:387-394.

[9] Pham TT, Ko SL (2010) A manufacturing model of an end mill using a five-axis CNC grinding machine. Int J Adv Manuf Tech, 48(5-8):461-72.

[10] Wang LM, Chen ZC, Li JF, Sun J (2016) A novel approach to determination of wheel position and 
orientation for five-axis CNC flute grinding of end mills. Int $J$ Adv Manuf Tech, 84(9-12):2499-2514.

[11] Chen FJ, Hu SJ, Yin SH (2012) A novel mathematical model for grinding ball-end milling cutter with equal rake and clearance angle. Int J Adv Manuf Tech, 63(1-4):109-116.

[12] Li GC, Sun J, Li JF (2014) Modeling and analysis of helical groove grinding in end mill machining. J Mater Process Tech, 214(12): 3067-3076.

[13] Tang F, Bai J, Wang XH (2014) Practical and reliable carbide drill grinding methods based on a five-axis CNC grinder. Int J Adv Manuf Tech, 73(5-8):659-667.

[14] Chen F, Bin HZ (2009) A novel CNC grinding method for the rake face of a taper ball-end mill with a CBN spherical grinding wheel. Int J Adv Manuf Tech, 41(9-10):846.

[15] Sartori S, Moro L, Ghiotti A, Bruschi S (2016) On the tool wear mechanisms in dry and cryogenic turning Additive Manufactured titanium alloys. Tribol Int, 105:264-273.

[16] Krishnaraj V, Samsudeensadham S, Sindhumathi R, Kuppan P (2014) A study on high speed end milling of titanium alloy. Procedia Eng, 97:251-257.

[17] Shi Q, Li L, He N, Zhao W, Liu XL (2013) Experimental study in high speed milling of titanium alloy TC21. Int J Adv Manuf Tech, 64(1-4):49-54.

[18] Amin AKMN, Ismail AF, Khairusshima MKN (2007) Effectiveness of uncoated WC-Co and PCD inserts in end milling of titanium alloy Ti-6Al-4V. J Mater Process Tech, 192-193(none):147-158.

[19] Yang P, Yao CF, Xie SH, Zhang DH, Tang DX (2016) Effect of Tool Orientation on Surface Integrity During Ball End Milling of Titanium Alloy TC17. Procedia CIRP, 56:143-148.

[20] Su HH, Liu P, Fu YC, Xu JH (2012) Tool Life and Surface Integrity in High-speed Milling of Titanium Alloy TA15 with PCD/PCBN Tools. Chinese J Aeronaut, 25(5):784-790.

[21] Wu CT, Chen CK, Tang YY (2001) Modeling and computer simulation of grinding the ball end type rotating cutter with a constant helical angle. P I Mech Eng B-J Eng, 215 (11):1581-1594.

[22] Tan DW, Guo WM, Wang HJ, Lin HT, Wang CY (2018) Cutting performance and wear mechanism of $\mathrm{TiB}_{2}-\mathrm{B}_{4} \mathrm{C}$ ceramic cutting tools in high speed turning of Ti6Al4V alloy. Ceram Int, 44(13):15495-15502.

[23] Yan DQ, Zhang DH, Luo M (2015) Optimization of Barrel Cutter for Five-axis Flank-milling Based on Approximation of Tool Envelope Surface. Comput Aided D \& App, 12(6):717-722.

[24] Luo M, Yan DQ, Wu BH, Zhang DH (2016) Barrel cutter design and toolpath planning for high-efficiency machining of freeform surface. Int J Adv Manuf Tech, 85(9):2495-2503.

[25] Chen T, Liu XL, Wang CH, Wang GY (2015) Design and fabrication of double-circular-arc torus milling cutter. Int J Adv Manuf Tech, 80(1-4):567-579. 